\title{
Referrals, Wait Times and Diagnoses at an Urgent Neurology Clinic over 10 Years
}

\author{
D.J. Wile, J. Warner, W. Murphy, A.L. Lafontaine, A. Hanson, S. Furtado
}

\begin{abstract}
Background: An urgent neurology assessment clinic was created at our institution to improve access to prompt neurological assessment, and has been in operation for over a decade. We assessed its timeliness and impact. Methods: The clinic database was examined retrospectively for trends in the volume and waiting time to assessments, neurologic diagnoses, and whether neurologic assessment changed patients' diagnoses. Before and after implementation, the frequency of emergency department neurology assessments and hospital admissions for neurological investigation were compared. Results: In the first decade, 25145 referrals were received; 12460 patients were accepted and assessed within an average of 3.8 working days. The most common problems seen included headache and seizure (20.2\% each). Overall, $44.6 \%$ of assessments resulted in a change to the referring diagnosis; this proportion varied by the type of problem seen (from $10.5 \%$ for seizures to $92.5 \%$ for psychiatric disturbances). From the pre- to post-opening periods, there were fewer emergency room neurological assessments (35.7\% reduction) and fewer hospital admissions for neurological investigation (4.4/week to 2.2/week, 50\% reduction). Conclusions: The urgent neurology clinic model at our institution has provided excellent service, including wait times of a few days, to a catchment of over two million Canadians for over a decade; clinic assessments have affected diagnoses and patient care.
\end{abstract}

RÉSUMÉ: Orientation d'un patient vers une clinique d'urgence en neurologie, temps d'attente et diagnostic au cours d'une période de 10 ans. Contexte : Une clinique d'urgence en neurologie a été établie pour offrir un accès plus rapide à une évaluation neurologique. Cette clinique est en opération depuis plus de 10 ans. Nous avons évalué son à-propos et son impact. Méthode : Nous avons examiné rétrospectivement la base de données de la clinique pour évaluer les tendances quant à l'achalandage et au temps d'attente pour une évaluation et un diagnostic neurologique et déterminer si l'évaluation neurologique avait modifié le diagnostic posé antérieurement chez le patient. Nous avons comparé la fréquence des évaluations neurologiques au département des urgences et les admissions à l'hôpital pour une évaluation neurologique avant et après l'ouverture de la clinique. Résultats : Pendant les dix premières années, nous avons reçu 25145 demandes de consultation ; 12460 patients ont été admis et évalués en moyenne dans les 3,8 jours ouvrables suivant le demande. Les problèmes les plus fréquemment rencontrés étaient la céphalée et les crises convulsives (20,2\% chacun). Au total, suite à 44,6\% des évaluations, le diagnostic a été modifié par rapport au diagnostic pour lequel le patient était référé. Cette proportion variait selon le type de problème rencontré (de 10,5\% pour les crises convulsives à 92,5\% pour les troubles psychiatriques). Il y a eu moins d'évaluations neurologiques au service des urgences après l'ouverture de la clinique (diminution de 35,7\%) et moins d'admissions à l'hôpital pour une évaluation neurologique (4,4 par semaine par rapport à 2,2 par semaine, soit une diminution de 50\%). Conclusions : Le modèle de clinique neurologique d'urgence à notre institution a fourni d'excellents services, dont un temps d'attente de seulement quelques jours, à un bassin de population de plus de 2 millions de Canadiens pendant plus de dix ans. Les évaluations cliniques ont influencé le diagnostic posé et les soins prodigués aux patients.

Can J Neurol Sci. 2014; 41: 260-264

Access to specialist care continues to be a concern for Canada's patients and primary care physicians. In an analysis from the 2010 Canadian Community Health Survey, the major barriers to access were unsurprising: availability (the ability to make referrals) and wait times ${ }^{1}$. Only $35.8 \%$ of general practitioners rate access to neurological care as "very good" or "excellent", 2 and for specialist wait times, Canada places 10th of 11 commonwealth countries, with $48 \%$ of patients waiting more than four weeks for their appointment. ${ }^{3}$ Efforts to address long wait times for neurological care in the United Kingdom and Australia have included email triage of neurological referrals ${ }^{4}$ and urgent assessment clinics. ${ }^{5-6}$ The Urgent Neurology Clinic (UNC) at the University of Calgary was created to improve access to urgent neurological assessment within the single payer model of public health care that predominates in Canada. The mandate of the program was to provide assessment, diagnosis and management of patients with new neurological problems within three working days.

The UNC has been functioning under this mandate for over a decade, accepting urgent referrals from any physician in our region. We undertook a retrospective analysis of the clinic referral and assessment databases for the first ten years of operation, seeking to determine the impact of the UNC on neurological patient care and resource use in our centre.

From the Department of Clinical Neurosciences (DJW, JW, WM, AH, SF), University of Calgary, Calgary, Alberta; Department of Neurology and Neurosurgery (ALL), McGill University, Montreal, Quebec, Canada.

Received May 30, 2013. Final Revisions Submitted September 5, 2013. Correspondence to: D.J. Wile, 12th floor Foothills Medical Centre, 140329 St NW Calgary, Alberta, T2N 2T9, Canada. Email: djwile@ucalgary.ca. 


\section{METHODS}

This study was approved by the University of Calgary Conjoint Health Research Ethics Board. Referrals to the UNC were accepted from physicians in the City of Calgary as well as other areas of Southern Alberta and Southeastern British Columbia, Canada, comprising an estimated catchment of more than 1.9 million people. ${ }^{7}$ The clinic was initially held at one hospital site, and expanded to a second hospital site after the eighth year of operation. Each site is staffed with one registered nurse and one booking clerk and holds five to seven half day clinics weekly.

All referrals were screened by two neurologists over the ten year study period (WM and $\mathrm{AH}$ ). Referred patients were declined (excluded) if they: 1) were deemed to need emergent assessment (the referring physician was advised to send the patient to the emergency room (ER)), 2) had recently seen a neurologist, 3) were current hospital inpatients, 4) had neurosurgical, traumatic or chronic neurological conditions, typical Bell palsy, or headache with normal cranial imaging 5) were referred for Workers' Compensation Board or second opinions. For the first nine years of clinic operation, declined referrals were returned to the referring physician with a list of general neurologists in our city with the shortest waiting lists. In the tenth year, our department initiated a central triage system for general neurology referrals; if UNC referrals are judged inappropriate by the triaging neurologist because they are not urgent, they are now often redirected as appropriate through this system to be seen by a neurologist, but not in the UNC. For those patients who were already followed by a neurologist, even for a different problem, referrals were declined and the patient's neurologist contacted directly.

\section{1) Clinic growth and sustainability}

Referral and assessment records for the first ten years of clinic operation were compiled in a clinic database after each patient's first visit to the UNC by the same experienced clinic nurse $(\mathrm{JW})$. We reviewed this database retrospectively for: annual growth in referrals, rates of rejected referrals, and referral source. Time from referral to assessment (in working days) was calculated. This information was available for the initial assessment, but not for followup visits.

A random selection of 300 family physicians was surveyed prior to UNC opening; 279 of the same physicians were surveyed one year after opening regarding satisfaction with regional access to urgent neurological assessment.

\section{2) Neurological Diagnoses in the UNC}

To characterize the different neurological problems encountered in the UNC, post-assessment diagnoses were listed and counted using Microsoft Excel (Redmond, WA). Etiologically related diagnoses were grouped together (e.g. "stroke", "intracerebral hemorrhage" grouped as "cerebrovascular disease"); diagnoses which could not be assigned an etiological category but based on the presenting features were also grouped (e.g. "paresthesias", "leg weakness" grouped as "sensorimotor disturbance", and "loss of consciousness" for those events without a specific diagnosis such as seizure or syncope; see Supplemental Table).
Referring (i.e. pre-assessment, referral diagnosis) and postassessment (neurologic diagnosis after initial neurologist's assessment) diagnoses were compared by one author (S.F.) for each patient visit and judged to be concordant or discordant. To be judged as discordant, referring and post assessment diagnoses needed to differ in a manner which was expected to impact care; for instance, many patients were referred with undifferentiated symptoms for neurological assessment, such as headache or numbness. For those cases in which a specific diagnosis was made (e.g. a referral for headache in which a diagnosis of migraine was made, or a referral with "hand numbness" diagnosed as "ulnar neuropathy at the elbow" or as "C8 radiculopathy") diagnoses were judged to be discordant. Similarly, those patients referred with "blackouts" with a postassessment diagnosis of "seizure" or "epilepsy" were judged to have discordant diagnoses. These data were tabulated on an annual basis for each diagnostic category.

\section{3) Resource use}

Emergency room charts listing neurological diagnoses were reviewed for two months prior to the clinic opening in September 2000 (September 1999, March 2000) and after opening (March 2001, September 2001). The number of ER patients with neurological diagnoses and number of referrals from family physicians and emergency physicians for neurology consults in the ER were compared before and after clinic opening. Data were also collected from a quality improvement initiative that ran in our centre prior to clinic opening (July 1 Sept 14, 2000) and after clinic opening (Sept 16, 2000 - Feb 28, 2001) to assess the reason for admission of neurological patients; the weekly number of hospital admissions 'for investigation' over these 10 weeks prior to and 22 weeks after UNC opening were compared. The proportion of patients requiring hospital admission was analyzed.

In the UNC, assessing neurologists ordered investigations for patients as deemed appropriate, and if studies were required urgently the neurologist or clinic staff would contact the diagnostic imaging, clinical neurophysiology or other department directly to expedite testing; obtaining such expedited testing is rarely a problem. When magnetic resonance imaging (MRI) was requested, the urgency of the request was recorded. We examined the number of referrals requested on an urgent $(<1$ week) basis; prior to clinic opening such referrals would often have resulted in emergency room consultation and hospital admission for investigation; we estimated the hospital admission days prevented using this information. We assessed the time elapsed before MRI completion for urgent MRI requests between 2006-2010, and for all requests in 2010 .

\section{RESULTS}

\section{1) Clinic growth and sustainability}

Annual referral and assessment data is summarized in Table 1. The number of referrals rose sharply $(33.8 \%)$ after the first year of clinic operation and have slowly grown over the intervening decade. The proportion of rejected referrals, based on exclusion criteria, remained at $40-50 \%$ annually. Family physicians in the community and emergency room physicians made the majority of referrals to the UNC, with a small 
Table 1: Annualized referral, assessment and investigation data

\begin{tabular}{|c|c|c|c|c|c|c|c|c|c|c|c|}
\hline Year & $\begin{array}{c}\text { Referrals } \\
\text { (\% growth) }\end{array}$ & $\begin{array}{c}\text { Referrals } \\
\text { rejected }(\%)\end{array}$ & $\begin{array}{c}\text { GP } \\
\text { Referrals } \\
(\%) \\
\end{array}$ & $\begin{array}{c}\text { ER } \\
\text { Referrals } \\
(\%) \\
\end{array}$ & $\begin{array}{c}\text { Specialist } \\
\text { Referrals } \\
(\%)\end{array}$ & $\begin{array}{c}\text { Unique } \\
\text { referring } \\
\text { MDs }\end{array}$ & Neurologists & $\begin{array}{c}\text { Assessments } \\
\text { (\% growth) }\end{array}$ & $\begin{array}{c}\text { Mean age } \\
(\mathrm{sd})\end{array}$ & $\begin{array}{l}\text { Days to } \\
\text { assessment } \\
\text { (sd) }\end{array}$ & $\begin{array}{c}\text { Pre/post } \\
\text { diagnoses } \\
\text { concordant }(\%)\end{array}$ \\
\hline 1 & 1629 & $39.2 \%$ & $931(57.2)$ & $656(40.3)$ & $40(2.5)$ & 406 & 12 & 948 & $42.9(16.3)$ & $3.6(2.6)$ & $542 / 943(57.5)$ \\
\hline 3 & $2298(5.5)$ & & $1186(51.6)$ & $1036(45.1)$ & $76(3.3)$ & 515 & 16 & $1248(-4.4)$ & $43.9(16.0)$ & $3.3(2.7)$ & $697 / 1246(55.9)$ \\
\hline 4 & $2596(13.0)$ & $48.7 \%$ & $1320(50.9)$ & $1205(46.4)$ & $69(2.7)$ & 516 & 18 & $1273(2.0)$ & $43.4(16.1)$ & $3.5(2.8)$ & $749 / 1274(58.8)$ \\
\hline 5 & $2560(-1.4)$ & $46.5 \%$ & $1242(48.5)$ & $1220(47.7)$ & $98(3.8)$ & 548 & 19 & $1222(-4.0)$ & $42.7(16.4)$ & $3.4(2.6)$ & $662 / 1215(54.5)$ \\
\hline 7 & $2870(7.6)$ & $51.2 \%$ & $1490(51.9)$ & $1273(44.4)$ & $105(3.7)$ & 592 & 20 & $1269(1.4)$ & $44.5(17.2)$ & $4.5(3.6)$ & $819 / 1259(65.1)$ \\
\hline 8 & $2722(-5.2)$ & $49.7 \%$ & $1388(51.0)$ & $1170(43.0)$ & $108(4.0)$ & 581 & 25 & $1185(-6.6)$ & $45.1(17.3)$ & $4.5(4.6)$ & $655 / 1152(56.9)$ \\
\hline 9 & $2858(5.0)$ & $49.5 \%$ & $1371(48.0)$ & $1343(47.0)$ & $140(4.9)$ & 718 & 29 & $1322(11.6)$ & $46.1(17.4)$ & $3.8(3.7)$ & $612 / 1314(46.6)$ \\
\hline 10 & $2766(-3.2)$ & $46.6 \%$ & $1389(50.2)$ & $1300(47.0)$ & $136(4.9)$ & 734 & 27 & $1436(8.6)$ & $45.5(17.4)$ & $3.8(3.8)$ & $658 / 1413(46.6)$ \\
\hline Total & 25145 & $46.1 \%$ & $12922(51.4)$ & $11313(45.0)$ & $940(3.7)$ & 2243 & & 12460 & $44.1(16.8)$ & $3.8(3.3)$ & $6559 / 11841(55.4$ \\
\hline
\end{tabular}

GP: General practitioner; ER: Emergency room (physician)

proportion (3.7\%) referred by specialists. There were 2243 individual physicians that referred patients to the UNC; of these 855 referred three or more patients.

In total, in the ten years of initial clinic operation, 25145 referrals were received, and 12460 of these patients were assessed in the UNC. Mean patient age has remained consistent, with an overall mean of 44.1 years (standard deviation (sd) 16.8).

The UNC visits took place, on average, 3.8 working days after referral (sd 3.3 days, interquartile range 2-5 days). Fiftyseven percent of patients $(\mathrm{N}=7072)$ met the UNC referral mandate of assessment within three working days; the patients not meeting the mandate were assessed on average within 6.3 days (sd 3.5). Average patient age was 44.1 (sd 16.8); annualized demographics are shown in Table 1 . Of 300 surveys sent to regional family physicians, 60 were returned (20\% response rate); of 279 surveys distributed one year after clinic opening, 59 were returned $(21.1 \%$ response rate). The proportion of

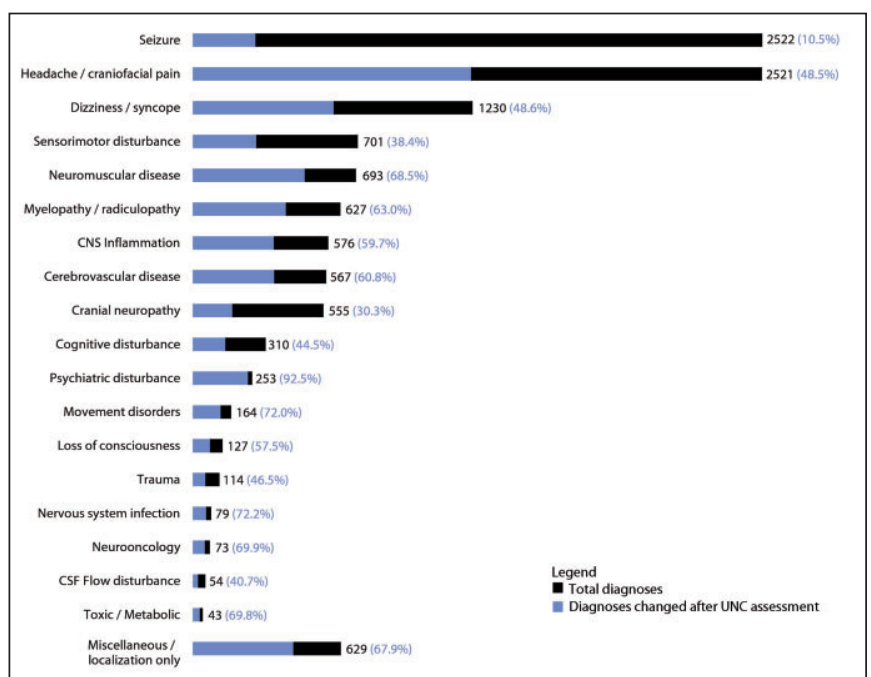

Figure: Diagnoses seen. Graphical representation of neurological diagnoses seen as judged after neurologist assessment. The proportion of patients originally referred with an alternate diagnosis is shown in blue. Labels indicate total number (percent changed). respondents reporting dissatisfaction with obtaining urgent neurology assessments was reduced between pre-opening $(83.3 \%)$ and post-opening $(41.9 \%)$ periods, while those reporting they were satisfied or very satisfied rose from $11.7 \%$ to $43.6 \%$.

\section{2) Neurologic Diagnoses in the UNC}

Diagnoses made after initial assessment by the UNC neurologists are summarized in the Figure; they consisted largely of seizure, headache and craniofacial pain, as well as varied causes of dizziness and syncope. 11919 of the 12460 UNC assessments had pre- and post-visit diagnosis data suitable for diagnosis categorization and comparison for concordance. Of these, 6559 (55.4\%) were concordant (i.e. the referral diagnosis and neurologist's diagnosis agreed) and 5282 (44.6\%) were discordant (i.e. UNC visit resulted in a significant change in neurologic diagnosis); this proportion was relatively stable every year. The rate of discordant pre- and post-assessment diagnoses varied considerably by diagnostic categories, from more than $70 \%$ (psychiatric disturbances, nervous system infections, movement disorders) to $30 \%$ or less (cranial neuropathy, seizures).

\section{3) Resource use}

Discharge records from the emergency department showed fewer emergency room neurological assessments after clinic opening (45 vs. 70, $35.7 \%$ reduction; Table 2). The quality improvement program determined a rate of 4.4 weekly neurological admissions 'for investigation' in the period prior to clinic opening and 2.2 weekly admissions in the period after opening ( $50 \%$ reduction). The assessing neurologist deemed that hospital admission was indicated for 250 patients (2.0\%) over ten years; the annual proportion of patients requiring admission remained stable.

Over ten years of clinic operation, 6829 (54.8\%) of patients assessed in UNC were subsequently booked for MRI, 1204 (9.7\%) for computed tomogram (CT) head, 3783 (30.4\%) for electroencephalogram (EEG), and $925 \quad(7.4 \%)$ for electromyelogram (EMG) / nerve conduction studies. One 
hundred and sixty MRI scans were requested for less than seven days; 68 of these occurred between year 6 and 10 of clinic operation and could be assessed for time to test completion. On average, these tests were completed within 7.0 days (sd 5.4 days) and 45 of these $(66.1 \%)$ were performed within the requested period; the remaining tests were performed an average of 5.0 days late (sd 6.3 days). Seven hundred and twenty eight MRI requests from the tenth clinic year were reviewed. The mean requested time for these MRI scans was within 44 days (sd 35 days), and MRI was performed on average 61.2 days after requisitioning (sd 66.6 days). 323 tests (44\%) were performed within the requested period, 353 were performed an average of 47.1 days late (sd 61.7 days), 29 requested tests were not performed due to cancellation and 22 were performed out of province or by a private provider.

\section{Conclusions}

The UNC at the University of Calgary has been successful in meeting a clinical need for urgent neurological assessment, and in maintaining an excellent waiting time (3.8 working days) for the large volume of patients who met clinic admission criteria. The UNC is unique within our catchment area of more than 1.9 million Canadians, which has grown by $22.9 \%$ in the study period. $^{7}$

More than 25000 referrals have been made by more than 2000 different physicians in this time frame, and more than 12000 patients seen. The UNC has grown from one to two hospital sites in our city, each with five to seven weekly half day clinics, and from 12 to 27 neurologists. The number of new assessments continues to increase annually, with attendant demand on clinic resources. In spite of this gradual rise in referrals, the rejection rate has not diminished, and ongoing work is required to direct non-urgent referrals to general neurology clinics and others to our local stroke prevention clinic as appropriate, and to educate referring physicians about UNC referral criteria. A central triage system for general neurology referrals was initiated in the tenth year of UNC operation; prior to this (during the study period) general neurology referrals outside of UNC would take up to one year to be seen (A Hanson, personal communication July 2013). It is our hope that the central triage process will streamline assessments for non-urgent neurological problems; general neurology referrals not appropriate for UNC are now triaged for assessment in either less than 4 weeks, 4-12 weeks, or more than 12 weeks (KD Busche, personal communication July 2013).

Outpatient services like UNC may help relieve burden on hospital resources ${ }^{5}$ and save emergency department bed time ${ }^{6}$. Avoidable admissions to hospital constitute a considerable healthcare expense, and in some studies have comprised up to $26 \%$ of neurological admissions; of these nearly $1 / 3$ may be appropriate for outpatient assessment. ${ }^{8}$ Despite this, there are few descriptions of such services in the literature. Specialized clinics for common neurological presentations such as a "first seizure" clinic can streamline care for such patients and result in faster confirmatory diagnostic testing and treatment of patients where appropriate..$^{9,10}$

Though it is difficult to quantify cost effectiveness in this retrospective study, we speculate that the UNC has had a positive impact on acute care resource use in our centre. For instance,
Table 2: Pre and post clinic resource use

\begin{tabular}{ccc}
\hline Acute care resource use & Pre & Post \\
\hline ER visits with neurological diagnosis & $544^{*}$ & $503^{* *}$ \\
UNC referrals from ER & $0^{*}$ & $54^{* *}$ \\
GP referral to ER for neurology consult & $23^{*}$ & $5^{* *}$ \\
ER referral for neurology consult & $47^{*}$ & $40^{* *}$ \\
& & $45^{* *}$ \\
Total ER neurology consults & $70^{*}$ & \\
Neurology admissions for investigation & $4.4 / \mathrm{wk} \#$ & $2.2 / \mathrm{wk} \#$ \\
\hline
\end{tabular}

*Sep 1999, Mar 2000; **Mar 2001, Sep 2001; \#Jul 1-Sep 14, 2000; \#\#Sep 16, 2000-Feb 28, 2001. ER: Emergency room, UNC: Urgent Neurology Clinic, GP: General practitioner

prior to UNC opening, when ambulatory patients were referred to on-call neurologists and thought to need urgent assessment, they likely would have been referred to the emergency department, and those requiring urgent investigations such as MRI would often have been admitted to hospital. On call neurologists can now arrange prompt outpatient assessment for many such patients. The rate of patients requiring hospital admission after UNC assessment for emergent problems remained low at around $2 \%$, indicating that few emergency cases were inappropriately triaged to UNC.

In our centre, problems which most often are referred for urgent assessment and which meet referral guidelines include seizure, headache and dizziness. The Calgary Stroke Prevention Clinic provides urgent assessment for transient ischemic attack and stroke in the same geographical region, resulting in some overlap in that such patients can be referred to either clinic; cerebrovascular disease is thus likely underrepresented in our data.

Nearly half of our patients' referring and post-assessment diagnoses differed. Some neurological problems in our series were more commonly referred with alternate diagnoses, particularly psychiatric disturbances such as conversion disorder, nervous system infections, and movement disorders. Though these categories had small overall numbers of assessments, two of the three largest categories (headache / craniofacial pain, dizziness / syncope) had alternate referring diagnoses in nearly half of patients, whereas those patients with seizure were less likely to have a change in diagnosis after UNC assessment. We speculate that these patient groups benefit from prompt neurological assessment in different ways; for patients with seizure, arrangement of prompt diagnostic testing and ongoing followup may be the primary benefit, and for others urgent clinical evaluation may change the provisional diagnosis and redirect the course of investigation and treatment (e.g. for conversion disorder). The rates of disagreement in referring diagnosis and neurologists' diagnosis were comparable to those in previous reports. ${ }^{11,12}$ The UNC at the University of Calgary has proven feasible, efficient, and highly valued by referring physicians in our centre, and is regarded as a valuable teaching environment for medical students and residents. Though response rates were low with initial physician satisfaction surveys they indicated that physicians were satisfied that access 
to neurological care had improved as a result of clinic opening. Informal feedback from patients and physicians continues to be positive.

Our study has shown that it is possible to sustain the effort of running such a clinic over a long period of time and to maintain this clinic with a high standard of care. Expedited investigations including neuroimaging and neurophysiological studies were feasible in our model and there were few referrals requiring redirection to acute care for hospital admission. This model of an urgent assessment clinic may be effective in reducing the burden of emergency department assessments and hospital admissions for neurological investigation.

\section{ACKNOWLEDGMENT}

The authors thank the neurologists and trainees who have worked and continue to work in the Urgent Neurology Clinic as well as the support of the Division of Neurology and Department of Clinical Neurosciences for the clinic.

\section{REFERENCES}

1. Harrington DW, Wilson K, Rosenberg M, Bell S. Access granted! Barriers endure: determinants of difficulties accessing specialist care when required in Ontario, Canada. BMC Health Serv Res. 2013;13:146-55.

2. National physician survey: results for family physicians by certification, sex, and age [Internet]. Ottawa ON: College of Family Physicians of Canada, Canadian Medical Association, Royal College of Physicians and Surgeons of Canada. c2010 [cited 2012 Jul 17]. Available from: http://www.national physiciansurvey.ca.
3. Schoen C, Osborn R, Squires D, Doty MM, Pierson R, Applebaum S. New 2011 survey of patients with complex care needs in eleven countries finds that care is often poorly coordinated. Health Aff. 2011;30(12):2437-48.

4. Patterson V, Humphreys J, Chua R. Email triage of new neurological outpatient referrals from general practice. J Neurol Neurosurg Psychiatr. 2004; 75:617-20.

5. Robertson NP, Shaunak S, Compston DAS. Urgent neurology outpatient referrals from primary health care physicians. Q J Med. 1998;91:309-13.

6. Ahmed RM, Green T, Halmagyi GM, Lewis SJ. A new model for neurology care in the emergency department. Med J Aust. 2010; 192:30-2.

7. GeoSearch 2011 Census - Statistics Canada Catalogue no. 92-142XWE [Internet]. Ottawa ON: Statistics Canada. c2011 [cited 2013 Feb 28]. Available from: http://geodepot.statcan.gc.ca/ GeoSearch2011-GeoRecherche2011/GeoSearch2011GeoRecherche2011.jsp.

8. Més-Sesé G, Plaza-Macías I, González-Caballero G, et al. [An analysis of avoidable admissions to a neurology service]. Rev Neurol. 2006;43(12):714-18.

9. Anang J, Tellez-Zenteno JF. Single unprovoked seizure: wait time to full medical assessment, does it matter? Neurol Bull. 2012;4: $1-11$.

10. Hernandez-Ronquillo L, Waterhouse K, Hunter G, Moien-Afshari F, Tellez-Zenteno JF. Outcomes in a single seizure clinic: a prospective Canadian study. Epilepsia. 2013;54(Suppl 3):38.

11. Moeller JJ, Kurniawan J, Gubitz GJ, Ross JA, Bhan V. Diagnostic accuracy of neurological problems in the emergency department. Can J Neurol Sci. 2008;35:335-41.

12. Chapman FA, Pope AE, Sorensen D, Knight RSG, Al-Shahi Salman R. Acute neurological problems: frequency, consultation patterns and the uses of a rapid access neurology clinic. J R Coll Physicians Edinb. 2009;39:296-300.

\section{Supplemental Table 1 : Diagnosis categorization}

\begin{tabular}{|c|c|}
\hline Category & Elements included \\
\hline Seizure & Seizure, Epilepsy, Sz, Convulsions, Miscellaneous epilepsy syndromes \\
\hline $\begin{array}{l}\text { Headache / craniofacial } \\
\text { pain }\end{array}$ & $\begin{array}{l}\text { Migraine, Headache, H/A, HA, Thunderclap, Exertional, Occipital neuralgia, Tension, Facial pain, Coital/orgasmic, Cluster, Hemicrania, Trigeminal neuralgia, Post-LP / } \\
\text { Low CSF, Cephalalgia, Glossopharyngeal, Cranial neuralgia, Miscellaneous cranial / facial pain }\end{array}$ \\
\hline Dizziness / syncope & Syncope, Dizziness, Vertigo, BPPV, Vestibular, Vasovagal, Lightheaded, Faint, Labyrinthitis, Meniere, Miscellaneous descriptive diagnoses \\
\hline Sensorimotor disturbance & Numbness, Weakness, Paresthesia, Dysesthesia, Sensory, Foot drop, Hemiparesis, Miscellaneous sensorimotor disturbances \\
\hline Neuromuscular disease & $\begin{array}{l}\text { Radial, Median, Ulnar, Peroneal, Plexopathy, Neuropathy, ALS / MND, Guillain Barré, Myopathy, Mononeuritis multiplex, Myasthenia gravis, Brachial neuritis, } \\
\text { Entrapment / compression neuropathy, Miscellaneous neuromuscular disease }\end{array}$ \\
\hline Myelopathy / radiculopathy & $\begin{array}{l}\text { Myelopathy, Radiculopathy, Cauda equina syndrome, Conus medullaris syndrome, Spine (excluding demyelination), Root, Lumbar, Cervical, Thoracic, Cord, Disc, } \\
\text { Sciatica, Claudication, miscellaneous disorders of spine / spinal nerve roots }\end{array}$ \\
\hline CNS Inflammation & Multiple sclerosis, optic neuritis, transverse myelitis, CNS inflammation, miscellaneous CNS inflammatory disorders \\
\hline Cerebrovascular disease & $\begin{array}{l}\text { Stroke, TIA, Aneurysm, Amaurosis, Vascular (cerebral only), Hemorrhage / SAH / SDH / hematoma, Infarct, Ischemia, Vertebrobasilar Insufficiency, Basilar, Vasculitis, } \\
\text { Thrombosis, Artery, Dissection, CVA, Miscellaneous cerebrovascular disease }\end{array}$ \\
\hline Cranial neuropathy & $\begin{array}{l}\text { Cranial Nerve, Trigeminal neuropathy, Bell palsy, Optic neuropathy, Facial numbness, Cranial palsy, Giant cell arteritis / temporal arteritis, Diplopia, Horner syndrome, } \\
\text { Anisocoria, Ptosis, Dysphagia, Dysarthria, internuclear ophthalmoplegia, Ramsay Hunt syndrome / Tolosa Hunt syndrome, Mydriasis, Miscellaneous cranial } \\
\text { neuropathies }\end{array}$ \\
\hline Cognitive disturbance & $\begin{array}{l}\text { Confusion, Alzheimer, Amnesia, transient global amnesia, Aphasia, Encephalopathy, MCI / cognitive decline, Memory problem, Pseudobulbar / emotional incontinence, } \\
\text { miscellaneous cognitive disturbances }\end{array}$ \\
\hline Psychiatric disturbance & $\begin{array}{l}\text { Stress, Anxiety, Depression, Conversion, Panic, Somatization, Somatoform, Hyperventilation, Functional, Dissociation, Nonepileptic attacks, Miscellaneous psychiatric } \\
\text { diagnoses }\end{array}$ \\
\hline Movement disorders & $\begin{array}{l}\text { Parkinsonism, Parkinson Disease, Ataxia, Tremor, Restless leg syndrome, dystonia, hemifacial spasm, blepharospasm, Myoclonus, Tics, Miscellaneous movement } \\
\text { disorders }\end{array}$ \\
\hline Loss of consciousness & Loss of consciousness, blackout, unresponsiveness \\
\hline Trauma & Concussion, postconcussive syndrome, trauma, head injury \\
\hline Nervous system infection & Encephalitis, Meningitis, Zoster, Shingles, Herpes, Postviral, West Nile Virus, abscess, neurocysticercosis, Miscellaneous infections \\
\hline Neurooncology & Tumor, Meningioma, Metastasis, Leptomeningeal metastasis / carcinomatosis, Paraneoplastic, Mass, Miscellaneous neoplasms \\
\hline CSF Flow disturbance & Pseudotumor, Hydrocephalus, Papilledema, Intracerebral pressure, Shunt, Miscellaneous CSF flow disturbances \\
\hline Toxic / Metabolic & Metabolic, Hypoglycemia/hypokalemia/hyponatremia, thyroid, Hepatic, Alcohol, Wernicke's encephalopathy, medication, Miscellaneous toxic / metabolic disorders \\
\hline $\begin{array}{l}\text { Miscellaneous / localization } \\
\text { only }\end{array}$ & $\begin{array}{l}\text { Myofascial, Musculoskeletal, Soft tissue, Fatigue, Unsteadiness / imbalance, Collapse, Spell, Incontinence, Vision disturbance, Back \& limb pain, Transient nonspecific } \\
\text { symptoms, Problems "not yet diagnosed", "Lesion" in specified location, "no neurological diagnosis", Sleep disturbances, Narcolepsy/cataplexy, Hemianopia, } \\
\text { Miscellaneous / otherwise noncategorizable }\end{array}$ \\
\hline
\end{tabular}

Postassessment diagnoses listed by the assessing neurologist were categorized in the above manner for inclusion in our analysis (see Figure 1). 